\title{
TTR
}

Traduction, terminologie, re?daction

\section{Traduire le « moult prouffitable » : Jacques Legrand (vers 1400) et la traduction pédagogique}

\section{Claire le Brun}

Volume 6, numéro 1, 1er semestre 1993

L'Histoire en traduction

URI : https://id.erudit.org/iderudit/037137ar

DOI : https://doi.org/10.7202/037137ar

Aller au sommaire du numéro

Éditeur(s)

Association canadienne de traductologie

ISSN

0835-8443 (imprimé)

1708-2188 (numérique)

Découvrir la revue

Citer cet article

le Brun, C. (1993). Traduire le « moult prouffitable » : Jacques Legrand (vers

1400 ) et la traduction pédagogique. TTR, 6(1), 27-60.

https://doi.org/10.7202/037137ar d'utilisation que vous pouvez consulter en ligne.

https://apropos.erudit.org/fr/usagers/politique-dutilisation/ 


\section{Traduire le «moult prouffitable»: Jacques Legrand (vers 1400) et la traduction pédagogique}

\section{Claire le Brun}

Pourquoi examiner le cas de Jacques Legrand dans une réflexion sur l'histoire de la traduction? Universitaire du début du $\mathrm{XV}^{\mathbf{e}}$ siècle, il a effectué pour un public laïc une traduction-adaptation en français de ses propres écrits didactiques en latin. Son Archiloge Sophie, qui «principalment parle de Sophie, autrement dicte sapience" ${ }^{1}$, explique l'auteur, se veut un compendium de toutes les sciences. La traduction de traités savants en vernaculaire n'est pas exceptionnelle à la fin du Moyen Âge. Nicole Oresme (mort en 1381) a largement ouvert la voie avec ses traductions d'Aristote et ses propres traités écrits successivement en latin et en français ${ }^{2}$. L'Archiloge Sophie présente cependant un

1. Texte édité par E. Beltran, l'Archiloge Sophie et le Livre de bonnes meurs (Paris, Champion, 1986), p. 25. Toutes les citations (les pages sont indiquées entre parenthèses dans le corps de l'article) de l'Archiloge Sophie renvoient à cette édition.

2. Traductions d'Aristote: $l^{\prime}$ Éthique d Nicomaque (1370), la Politique (1374); l'Économique (1374) et $\mathrm{Du}$ Ciel et du monde (1377), faussement attribués à Aristote. Auto-traductions de traités latins: Traictié du commencement et premiere invention des monnoies (vers 1360) et Livre de divinacion (1366). Voir M. Clagett, "Oresme, Nicole», The Dictionary of Scientific Biography (1970- 
intérêt particulier comme adaptation française et pour le français, l'une des plus anciennes du genre, des arts du trivium, qui ont transmis au Moyen Âge une théorie du langage héritée de l'Antiquité: grammaire, logique et rhétorique. La présente étude soulève trois questions principales: En quoi la décision de traduire modifie-t-elle la finalité du texte? Quelle fraction de l'héritage classique est jugée traduisible par l'auteur et dans quel cadre théorique latin organise-t-il son enseignement en français? Quelle conception de la traduction reflètent les techniques utilisées dans l'Archiloge Sophie?

\section{Statut et fonction de la traduction dans l'œuvre de J. Legrand}

\subsection{Vie et œuvre de Jacques Legrand}

Jacques Legrand est né aux environs de $1360^{4}$. Son activité s'est surtout déployée à Paris, sous le règne troublé de Charles VI, le

1980); A. D. Menut, Maistre Nicole Oresme. Le Livre de Politiques d'Aristote, Transactions of the American Philosophical Society, New series, LX, 6 (1970); P. Chavy, Traducteurs d'autrefois. Moyen Âge et Renaissance (Paris-Genève, Champion-Slatkine, 1988, t. 2), pp. 1021-1024. Sur la rédaction bilingue aux XIV et $\mathrm{XV}^{\mathrm{e}}$ siècles, voir $\mathrm{A}$. Vernet, «les Traductions latines d'œuvres en langues vernaculaires au Moyen Âge», Traduction et traducteurs au Moyen Age, G. Contamine, dir. (Paris, CNRS, 1989), pp. 225241.

3. Avant J. Legrand, on connait un cas, isolé, de traduction de la rhétorique latine en français. Il s'agit de la traduction par Jean d'Antioche du De inventione de Cicéron et de l'Ad Herennium (1282), conservée par un seul manuscrit, Musée Condé 590. Voir L. Delisle, "Maître Jean d'Antioche, traducteur, et frère Guillaume de Saint-Etienne, hospitalier", Histoire littéraire de la France, XXXIII (1906), pp. 1-40; P. Chavy, Traducteurs d'autrefois. Moyen Age et Renaissance $(1988$, t. 2), p. 764.

4. Sur la biographie de J. Legrand, voir l'étude la plus récente: $\mathrm{E}$. Beltran, "Jacques Legrand, O.E.S.A. Sa vie et son œuvre", Augustiniana 30 (1974), pp. 132-160 et 387-414. 
roi fou, et au moment d'une reprise des hostilités de la guerre de cent ans ${ }^{5}$. Entré dans l'ordre des Ermites de Saint-Augustin, il étudie la philosophie et la théologie. La plus ancienne notice biographique de Legrand se trouve dans une chronique de cet Ordre ${ }^{6}$. Plusieurs des œuvres latines de Legrand renvoient à son cursus universitaire ou à sa charge professorale ${ }^{7}$. Cependant, c'est surtout à l'extérieur du monde universitaire que Legrand a connu la célébrité: il s'est illustré à trois titres, comme humaniste, comme orateur, comme homme politique.

L'humaniste: Jacques Legrand naît au moment où se forme le premier groupe d'humanistes français, admirateurs de Pétrarque, gravitant autour de la cour papale d'Avignon et de Paris: Gontier Col, Nicolas de Clamanges, Jean de Montreuil ${ }^{8}$. Jacques Legrand ne semble pas avoir fait partie d'un cercle, mais la correspondance de Jean de Montreuil montre qu'il tenait ce

5. Sur le contexte historique, voir E. Jarry, la Vie politique de Louis de France, duc d'Orléans, 1372-1407 (Genève, Slatkine-Megariotis Reprints, 1976; $1^{\text {tre }}$ éd. 1889); R. Cazelles, Société politique, noblesse et couronne sous jean le Bon et Charles $V$ (Genève-Paris, Droz, 1982); B. Schnerb, les Armagnacs et les Bourguignons. La maudite guerre (Paris, Perrin, 1988).

6. Ambrosius de Cora (Coriolanus). Defensorium ordinis Augustianorum cum chronica sacrat. Ordinis fratrum Eremitarum $S$. Augustini (Rome, 1481).

7. Principium super Bibliam, leçon inaugurale prononcée en 1401, éd. E. Beltran, "Jacques Legrand prédicateur", Analecta Augustiniana 30 (1967), pp. 148-209; Postilla tam litteralis quam mystica super librum Genesis, inédit; Expositio in Psalmos; Lectura super quattuor libros Sentenciarum. Pour la liste des manuscrits, voir E. Ypma, «les Auteurs augustins français Jacobus Magnus)", Augustiniana 20 (1970), pp. 348-355.

8. Voir A. Combes, Jean de Montreuil et le chancelier Gerson (Paris, Vrin, 1973). 
dernier en haute estime et admirait son éloquence? Legrand a laissé un recueil de citations d'auteurs classiques: Aristotelis, Seneca, Boecii dicta communiora ${ }^{10}$. Son ceuvre la plus diffusée, le Sophilogium, est présentée comme un florilège des auteurs antiques, surtout les poètes.

L'orateur, le prédicateur: Prédicateur de cour, réputé pour son éloquence et son audace, il n'hésite pas, au cours de sermons d'apparat, à reprocher à la reine Isabeau de Bavière ses liaisons scandaleuses et au roi son avidité et sa corruption ${ }^{11}$.

L'homme politique: Entre 1409 et 1414, Jacques Legrand est l'un des principaux conseillers du parti des Armagnacs. Il est chargé de plusieurs missions auprès des ducs de Bretagne, de Berry, et auprès du roi d'Angleterre, Henri IV ${ }^{12}$.

Ces aspects de la biographie du moine augustin rendent manifeste son insertion dans la sphère séculière. Les bribes de

9. J. Legrand ne semble avoir répondu qu'une seule fois aux nombreuses lettres de Jean de Montreuil ("cumulatis meis scriptionibus», lettre 179, Opera, vol. I Epist., éd. E. Ornato, Turin, 1963). Sur les rapports de J. Legrand et de J. de Montreuil, voir A. Combes, "Jacques Legrand, Alfred Coville et le Sophilogium», Augustiniana 7 (1957), pp. 327-348 et 493-551 et Augustiniana 8 (1958), Pp. 129-163; E. Beltran (1974).

10. Mss Paris, Arsenal 481, f. 1-40; Würzburg, Univ. Bibl. M.ch.q.3, f. 116-145.

11. Voir les récits de Guillebert de Metz (Description de la ville de Paris au XV' siècle, éd. Le Roux de Lincy-Tisserands dans Paris et ses historiens, Paris, 1862), de Jean Juvénal des Ursins (Chroniques, éd. Michaud et Poujalat, Paris, 1836) et du Religieux de Saint-Denis.

12. Voir Enguerrand de Monstrelet, Chronique, éd. Douët d'Arcq, (Paris, Verdière, 1826-1827, t. II), p. 241. 
chronique $^{13}$ ou de correspondance ${ }^{14}$ sur lesquelles se fonde notre connaissance de l'homme aident à comprendre la fonction de ses écrits, notamment les écrits français qui nous intéressent ici.

\subsection{Les œuvres de Legrand et leurs destinataires}

Les trois œuvres majeures de Jacques Legrand sont le Sophilogium, l'Archiloge Sophie et le Livre de bonnes meurs, toutes trois écrites dans les premières années du $X V^{e}$ siècle ${ }^{15}$. Les deux œuvres en français sont des amplifications du Sophilogium latin. Le Sophilogium est dédié à Michel de Creney, évêque d'Auxerre. Dans une lettre-préface, l'auteur explique ses intentions:

... presentem libellum ex dictis poetarum precipue compegi quem Sophilogium interpretor eo quod principali intentio est inducere legentis animum ad amorem sapientie ${ }^{16}$.

Le Sophilogium est donc un recueil de citations d'auteurs antiques destiné à inspirer au lecteur l'amour de la sagesse. Il comporte trois parties principales: l'amour de la sagesse, l'amour des vertus, les différents états de vie. Dans les adaptations françaises, l'AS traite de la première partie, l'amour de la sagesse, et le Livre de bonnes meurs des deux autres. Alors que le Sophilogium est dédié à un ecclésiastique, $\mathrm{l}^{\prime} A S$ et le Livre de bonnes meurs sont dédiés à des laïcs, à des princes, respectivement Louis d'Orléans, frère du roi et chef du parti des Armagnacs, et Jean de Berry,

13. Cf. notes 4 et 6 .

14. Cf. note 9 .

15. Sur la datation des trois ceuvres et leurs rapports réciproques voir Beltran (1974) et Beltran (1986), pp. 11-12. Le titre Archiloge Sophie sera désormais abrégé en $A S$.

16. Ms Paris, B.N. lat. 3235, f. 1d. Cité par Combes (1957), p. 342. E. Beltran a terminé une édition critique du Sophilogium qui est encore inédite. 
oncle de Charles VI. Par la traduction, le texte change de fonction: il entre dans la catégorie des Miroirs du prince.

\subsection{Les Miroirs du prince médiévaux}

Il importe de présenter en quelques mots les Miroirs du prince médiévaux. Ce genre didactique a été florissant de la Renaissance carolingienne au $\mathrm{XVII}^{\mathrm{e}}$ siècle, soit en latin soit en langue vernaculaire $^{17}$. Le plus célèbre, et le plus lourd d'influence, est le Policraticus de Jean de Salisbury $(1159)^{18}$, composé pour Thomas Beckett. Jean de Salisbury est l'auteur de la formule, qui deviendra adage à la fin du Moyen Âge: «Rex illiteratus quasi asinus coronatus» ${ }^{19}$. L'auteur de l'AS se réfère explicitement au Policraticus dont il traduit la célèbre formule: «Roy non lettré est comme asne couronné.» (p. 37) Les Miroirs du prince ont deux sujets principaux, dont l'un ou l'autre domine selon les traités: les vertus du prince idéal et l'art politique. Sous le règne de Charles VI, qui accède au trône à douze ans et présente peu après des symptômes de folie, le genre des Miroirs du prince connaît un regain de faveur. Citons les plus célèbres: le Songe du viel pelerin de Philippe de Mézières (1389), véritable programme politique, et le Livre du corps de policie de Christine de Pisan (1405). Si le Livre de bonnes meurs est en tout point conforme a la tradition éthique des Miroirs du prince, $l^{\prime} A S$ par contre me paraît occuper une place à part et même constituer un développement original du genre. L'AS repose en effet sur le credo de l'auteur en la sapience, sagesse acquise principalement à la fréquentation des auteurs antiques. Pour Legrand, $c^{\prime}$ est dans les sources antiques que les princes doivent étudier les sciences qui sont "vraies et prouffitables", selon les termes de l'auteur (p. 31). Le vrai -

17. Voir J. Krynen, Idéal du prince et pouvoir royal en France da la fin du Moyen Áge (1380-1440) (Paris, A. et J. Picard, 1981), pp. 5171.

18. Policraticus sive de nugis curialium et vestigiis philosophorum, éd. C.C.J. Webb (Oxford, 1909).

19. Policraticus, VI. 
exigence à la fois intellectuelle, éthique et religieuse - et le prouffitable, c'est-à-dire à la fois l'accessible et l'utile - exigence pédagogique -, constituent les critères du traducteur pour la sélection des contenus:

Et dois aviser que tu ne dois pas étudier sciences trop haultaines a ton entendement ne sciences deffendues, comme sont les sciences de nigromancie. (p. 50)

Dans son panorama des Miroirs du prince écrits sous le règne de Charles VI, Jacques Krynen (1981, pp. 60-62) examine le Livre de bonnes meurs et ignore l'AS. Il s'étonne alors qu'un homme si mêlé aux affaires politiques de son temps ait écrit un Miroir si archaïsant (p. 61). Selon nous, le texte de l'AS, de composition antérieure au Livre de bonnes meurs, porteur d'un optimisme humaniste, est véritablement novateur; il vise en effet à doter le prince de ressources expressives et persuasives en langue vernaculaire, à mettre à sa disposition le pouvoir de la parole.

\subsection{Le propos de l'auteur de l'Archiloge Sophie}

L'AS nous intéresse comme entreprise d'organisation et de présentation pédagogique d'une somme de connaissances héritées de la latinité. Le plan initial de l'auteur est ainsi annoncé: «Je parlerai de toutes les sciences dont Dieu m'a donné connaissance.» (p. 25) Dans l'état inachevé qui est conservé dans quatre manuscrits ${ }^{20}$, le texte, après une première partie sur l'amour de sapience, suit le plan des arts libéraux: trivium (grammaire, logique, rhétorique) et quadrivium (arithmétique, inachevée). L'un des arts du trivium domine les autres, en longueur et en intérêt pour notre propos. C'est la rhétorique, où nous voyons un passionnant exemple de traduction d'un genre,

20. Mss Paris, B.N. fr. 143 (anc. 6808); Paris, B.N. fr. 1508 (anc. 7570); Paris, B.N. fr. 214 (anc. 6268); Paris, B.N. fr. 24232 (anc. 660). E. Beltran prend le ms Paris, B.N. fr. 214 comme texte de base. Pour l'étude critique de la tradition manuscrite, voir Beltran (1986), pp. 20-22. 
d'un ensemble de règles, de codes et de concepts. À travers ce texte, s'observe le passage de l'art oratoire antique à ses avatars médiévaux ${ }^{21}$ : ars dictaminis, art versificatoria, ars predicandi débouchant chez Legrand sur une éloquence en langue vulgaire à portée séculière et politique.

Deux réflexions de l'auteur, dans l'épître dédicatoire, ont orienté notre lecture: (1) «Et ja soit ce que j'aye ce present livre en latin composé, comme il est dessus dit, touteffois plusieurs choses en françois, c'est assavoir en ce livre cy, je laisse comme pou prouffitables, lesquelles en latin sont assez necessaires, mais en français ne se peuent bonnement declairer.» (p. 25) Ce constat de perte d'information dans le transfert du latin à une langue vernaculaire est familier aux historiens de la traduction. L'intérêt du commentaire de Legrand est ailleurs. L'auteur, rédacteur bilingue, présente les disparités entre ses deux textes comme une décision pédagogique: certaines "choses" - sujets, concepts ou termes - n'ont pas d'utilité en français. Est-ce à dire dans la culture-cible, pour le destinataire, dans ce type de discours?

(2) «Et de fait en françois aucunes sont escriptes ou procès de ce livre, lesquelles de prime face se monstrent estranges; neantmoins le lisant les pourra bien entendre se il veult diligemment aviser.» (p. 25) Dans le même temps le traducteur a conscience d'innover, de faire reculer les frontières du dicible en français. Nous examinerons plus bas les procédés choisis pour introduire les nouveaux concepts et signifiants.

2. Jacques Legrand, diffuseur de la culture antique et traducteur d'une théorie latine

\subsection{Jacques Legrand, traducteur et rédacteur bilingue}

Le processus de traduction qui est mis en œuvre dans l'AS doit être entendu de deux façons:

21. Voir l'excellente étude de J. J. Murphy, Rhetoric in the Middle Ages. A History of Rhetorical Theory from Saint Augustine to the Renaissance (University of California Press, 1974). 
- traduction des autorités avec ses variantes d'explication, d'abrégé, d'amplification, d'adaptation sélective;

- reformulation, avec les mêmes variantes, de ses propres travaux en latin.

Les rapports entre le Sophilogium et les deux cuvres françaises ont été longuement discutés, notamment par Evencio Beltran, l'éditeur des textes ${ }^{22}$. Ce dernier qualifie les œuvres françaises de traduction-adaptation ${ }^{23}$. La partie du texte qui nous intéresse, la rhétorique, est un exemple patent d'amplification par rapport à l'original. La perspective de l'auteur est tout à fait différente ici puisqu'il s'agit d'organiser, à partir d'un cadre latin, un art de la parole en français.

Beltran a identifié les sources latines de l'AS, dont la majorité sont communes au Sophilogium et à $\mathrm{l}^{\prime} A S$. Pour une grande partie de la rhétorique, toutefois, il estime que Legrand a puisé à ses souvenirs de manuels scolaires, aussi difficiles à identifier qu'ils se ressemblent tous (Beltran, 1986, pp. 15-16). Dans l'optique qui est la nôtre, cette absence de sources certaines est d'une importance secondaire dans la mesure où il s'agit d'observer le travail de sélection/rejet de Legrand dans la tradition rhétorique latine classique et médiévale, plutôt que de faire une analyse comparative d'un original et de sa tradition. L'AS sera donc étudiée en regard de deux corpus:

- les traités et les manuels de rhétorique qui ont constitué la référence au Moyen Âge;

- les écrits de Legrand dans le domaine de la rhétorique latine.

22. Voir notes 1 et 15.

23. Sur la problématique de l'adaptation au Moyen Âge, voir D. Kelly, "Translatio studii: Translation, Adaptation, and Allegory in Medieval French Literature», Philological Quarterly 57 (1978), pp. 287-310; la préface de J. Beer dans Medieval Translators and Their Craft (Kalamazoo, Michigan, 1989), pp. 1-8. 


\subsection{Les traités et les manuels de rhétorique}

La rhétorique médiévale a eu pour "autorités» des traités comme la Rhétorique d'Aristote, le De oratore de Cicéron, l'Institutio oratoria de Quintilien et le tardif De differentiis topicis de Boèce. Cependant le modèle universellement lu et glosé a été l'ensemble constitué par le De inventione de Cicéron, cuvre de jeunesse, et un traité d'un maître anonyme du premier siècle av. J.C., et que tout le Moyen Âge a attribué à Cicéron, la Rhétorique à Herennius ${ }^{24}$. Les deux textes étaient connus comme la première et la seconde ou l'ancienne et la nouvelle rhétoriques de Cicéron. Dans sa présentation de la rhétorique, Legrand cite la vieille et la nouvelle rhétorique de Tulle, ainsi que les Topiques de Boèce. De toutes les cuvres que nous venons de mentionner, c'est le pseudo-Cicéron qui a eu l'influence la plus large et la plus durable. Le quatrième livre de l'Ad Herennium, consacré aux figures de style (exornationes verborum), a souvent été isolé de son contexte pour constituer un recueil autonome. Car les figures occupent une place de premier plan dans la rhétorique médiévale; omniprésentes, elles appartiennent également au domaine d'études de la grammaire. Les différents domaines de la rhétorique médiévale vont se constituer en sélectionnant et en développant certaines composantes de la rhétorique antique: la grammaire qui empiète sur la rhétorique en incluant l'étude stylistique des auteurs classiques (enarratio poetarum); l'ars dictaminis ou l'art d'écrire les lettres officielles, dès le XI' siècle ; les arts poétiques (ars versificatoria, poetria) au XII siècle; les artes predicandi, au XIII ${ }^{e}$ siècle avec l'essor des Ordres mendiants ${ }^{25}$. Ce

24. Cf. Murphy (1974), pp. 3-42; C.S. Baldwin, Medieval Rhetoric and Poetic (to 1400) (New York, McMillan, 1928); J. O. Ward, «From Antiquity to the Renaissance: Glosses and Commentaries on Cicero's Rhetorican, in J.J. Murphy, dir. Medieval Eloquence (London-Los Angeles, University of California Press, 1978), pp. $25-66$.

25. Cf. Murphy, op. cit.; E. Faral, les Arts poétiques du XIr et du XIIF siecle (Paris, Champion, 1924); Th. M. Charland, Artes praedicandi (Paris-Ottawa, 1963). 
dernier art, qui marque un retour à la pratique orale de la rhétorique, est celui qui prépare le mieux la démarche de Jacques Legrand.

Cette théorie est transmise en latin. Cependant le XIV siècle voit l'apparition d'une théorie en français dans un domaine: les traités de versification, appelés traités de seconde rhétorique ou de rhétorique laie (laïque) ${ }^{26}$. L'Art de dictier d'Eustache Deschamps (1392) précède de quelques années l'AS. Comme nous le verrons plus bas, J. Legrand fait écho à cette rhétorique laie, mais dans un cadre de pensée différent: sa rhétorique française, à l'usage des laïcs elle aussi, se veut avant tout transmission autant qu'il est possible de l'héritage latin.

\subsection{CEuvres théoriques de Jacques Legrand en latin}

Dans la rhétorique de l'AS, Legrand adapte en français de courts traités écrits en latin pour des ecclésiastiques ou des notes personnelles. C'est le cas de son Introductorium sermocinandi ${ }^{27}$ dont le premier chapitre est consacré aux rimes et au rythme et de son Tractatus de arte memorandi ${ }^{28}$ dont il tirera douze règles de mémorisation pour l'AS.

\subsection{Jacques Legrand traducteur de 1'Ovidius moralizatus de Pierre Bersuire}

Toutes les sources de Legrand n'appartiennent pas au corpus de la rhétorique. L'auteur termine sa rhétorique par un recueil d'exempla pour orateurs en deux parties: exemples mythologiques tirés d'Ovide et exemples bibliques. On sait que les Métamorphoses d'Ovide ont été relues à la lumière chrétienne et interprétées litteraliter, naturaliter, historialiter, spiritualiter par

26. Cf. E. Langlois, Recueil d'arts de seconde rhétorique (Paris, Imprimerie nationale, 1902).

27. Édité en partie dans Beltran (1967), pp. 162-168.

28. Inédit. Ms Paris, Arsenal 542, ff. 76v-79v. 
des commentateurs médiévaux recherchant les quatre sens sous l'écorce de la fabula ${ }^{29}$. Legrand compose son recueil à partir du Repertorium morale de Pierre Bersuire (vers 1360), dont le livre XV est un commentaire moral et allégorique des Métamorphoses d'Ovide. L'examen de cette source permettra d'observer plus précisément une des techniques de traduction de Legrand.

\subsection{Position du traducteur devant la culture-cible}

Voilà donc explicité ce qu'il faut entendre, dans le cas de $l^{\prime} A S$, par traduction/adaptation de textes latins. Observons maintenant comment Legrand se situe par rapport aux deux systèmes linguistiques et aux deux cultures en présence.

L'état de diglossie des clercs médiévaux a été étudiée ${ }^{30}$. Legrand ne fait pas exception à la règle. Cependant le partage des activités intellectuelles qu'établit Legrand entre les deux systèmes linguistiques mérite que l'on s'y arrête quelque peu.

Dans le chapitre sur la grammaire (pp. 58-67), que nous ne pouvons étudier ici, J. Legrand définit cette science comme la description des trois grandes langues de communication: l'hébreu, le grec et le latin. La possibilité d'une grammaire du vernaculaire n'est pas évoquée. De la description des trois langues, il ressort clairement que le latin occupe la première place dans la hiérarchie de Legrand. L'hébreu est une langue "moult imparfaite», car elle compte peu de mots; le latin "parle plus proprement et plus parfaitement» et est plus «légier à apprendre» (p. 59). Quant au grec, il comporte cinq dialectes

29. Voir P. Demats, Fabula. Trois études de mythographie antique et médiévale (Genève, Droz, 1973); J. Engels, Etude sur l'Ovide moralisé (Groningen, 1943); E.K. Rand, Ovid and His Influence (Boston, 1925); J. Seznec, la Survivance des dieux antiques (Londres, 1940); trad.: The Survival of the Pagan Gods (Princeton, N.J., 1972).

30. Pour le cas du moyen français, voir S. Lusignan, Parler vulgairement (Vrin-Presses de l'Université de Montréal, 1986). 
alors que le latin est «tout un à tous» (pp. 62-64), malgré les variations phonétiques. La valorisation par Legrand de sa langue "professionnelle» est manifeste. Loin de lui le pessimisme de Roger Bacon sur les possibilités du latin ${ }^{31}$ ! Legrand est un usager enthousiaste du latin «tierce langue principale, de laquelle nous usons» (p. 64). Il y voit une langue riche et indispensable a la communication. Toutefois, il est intéressant de constater que cela ne l'empêche pas de poser le problème des besoins spécifiques du français et de remettre en cause sur certains points la dépendance du français vis-à-vis de la langue mère. Ainsi, après l'énumération des lettres latines, Legrand souligne, avec exemples à l'appui, que le $i$ et le $u$ latins sont insuffisants pour noter le $i$ et le $j$, le $u$ et le $v$, et dit s'opposer sur ce point à l'opinion reçue ${ }^{32}$. Il ressort de l'ensemble des réflexions disséminées dans le texte une attitude d'usager de deux systèmes linguistiques qui en maîtrise également les effets: Legrand est orateur en latin et en langue vernaculaire. Son aisance dans l'éloquence française est attestée par les très nombreux exemples qu'il dispense au destinataire du livre, et qui constituent autant de fragments de discours en prose rythmée ou en vers. Legrand assume le rôle de passeur, enrichissant le français de techniques éprouvées en latin, retenant de l'éloquence dite cicéronienne ce qui peut "prouffiter» à l'usager du vernaculaire.

31. Voir particulièrement $P$. Bourgain, «le Sens de la langue et des langues chez Roger Bacon", in G. Contamine, dir. Traduction et traducteurs au Moyen Ȧge, pp. 317-331.

32. «Et dient les communs gramairiens que elles sont soufisantes sanz autres lectres trouver; mais a mon avis les lectres latines seroient plus souffisantes se elles estoient differentes selon la difference des voix: car ce seroit chose prouffitable de avoir diverses manieres de escripre selon la diversité du parler." (p. 65) 
2.6. La rhétorique de $I^{\prime} A S$ : réagencement en français du $D e$ Inventione de Cicéron et de l'Ad Herennium avec greffe de la rhétorique laie

La rhétorique de l' $A S$ est une longue section (plus de quarante folios) dont l'économie n'est pas immédiatement perceptible. Les lectures rapides qui en ont été faites jusqu'à présent ont repéré d'emblée le plan, traditionnel dans les recueils de Colores rhetorici, des couleurs de parole et de pensée. J. Legrand écrit en effet dans l'introduction: "Pour la quelle rethorique entendre tu dois savoir que rhetorique contient deux manieres de couleurs.» (p. 85) S. Lusignan croit que la rhétorique de Legrand s'organise autour de ce catalogue; il voit dans l'AS une stylistique et la rattache à la tradition des arts poétiques, donc au domaine de l'écrit ${ }^{33}$. Quant à nous, nous la rattachons sans hésiter aux artes predicandi, aux arts de la parole, et nous y voyons, par la traduction en français et le changement de public-cible, un nouveau traité d'éloquence séculière, réactivation de l'éloquence antique. A cet égard, la réputation d'humaniste de Legrand n'est pas usurpée.

Mais venons-en à l'analyse de cette rhétorique comme adaptation française de la rhétorique de Tulle. Comme les autres sections importantes du livre, la rhétorique est introduite par un quatrain. Legrand utilise également les quatrains dans ses sermons latins pour introduire le thème ${ }^{34}$. Le quatrain résume la conception qu'a Legrand de la rhétorique:

Tu pues par rethorique ton propos colourer, Et toutes tes parolles par raison bien parer. Rhetorique langage fait par sa douceur plaire, Et scet les escoutans a soy oir actraire. (p. 84)

La rhétorique est donc un art de la parole (parole, escoutans) qui vise à la persuasion (a soy oir actraire) par des moyens esthétiques (colourer, parer). Plus loin J. Legrand distingue entre

33. Lusignan, op. cit., p. 182.

34. Cf. Beltran (1974): textes de sermons latins de J. Legrand. 
la logique, qui procède par arguments et la rhétorique, qui procède par persuasion. Legrand définit le domaine respectif des trois arts du langage par la distinction traditionnelle: artes recte, vere, bene dicendi: grammaire, parler congruemment; logique, parler vraiement; rhétorique, parler bel et plaisamment (p. 84). On remarquera que l'adverbe «bene» dont les signifiés renvoient dans l'esprit de l'auteur à des catégories morales et non esthétiques, a été traduit par un binôme: bel et plaisamment. Plus bas, l'auteur opposera le bien dire, correspondant aux couleurs de sentence et le beau dire, correspondant aux couleurs de pensée.

La principale prise de position de l'auteur, relativement à notre propos, c'est la traduisibilité de principe de la rhétorique: «Rhetorique est science moult prouffitable et necessaire non mie seulement en latin, mais en français et en tous langages.» (p. 85)

\subsection{Les couleurs de sentence et les couleurs de parole}

\subsubsection{Les couleurs de sentence}

Nous constatons d'abord que l'ordre traditionnel depuis l'Ad Herennium: figures de mots (exornatio verborum), tropes, figures de pensée (exornatio sententiarum) est inversé chez Legrand. L'auteur explique que le bien dire (sentence) doit précéder le beau dire (parole). L'inversion pourrait aisément s'expliquer par les scrupules d'un prédicateur plus soucieux de morale que d'esthétique. Mais, à y regarder de plus près, il y a plus qu'une simple interpolation.

L'auteur présente 13 couleurs de sentence et 13 couleurs de parole, contre les 64 figures de $\mathrm{l}^{\prime}$ Ad Herennium ${ }^{35}$. Dans les couleurs de sentence de Legrand, on cherchera vainement la liste

35. Les citations de l'Ad Herennium renvoient à l'édition Loeb: $A d$ C. Herennium Libri IV de ratione dicendi, éd. par T.E. Page et coll. (Harvard University Press et William Heinemann Ltd., 1954). Les pages seront placées immédiatement après les citations et indiquées de la manière suivante: (Ad Her., page). 
familière de l'Ad Herennium, fidèlement reprise dans les manuels médiévaux: distributio, licentia, diminutio, descriptio, divisio, etc. Ces figures relevaient des techniques de présentation et d'agencement des idées: exposition des faits, énumération, comparaison, dialogue, prosopopée, et de captatio benevolentiae. Legrand commence l'exposé des treize figures de pensée en indiquant que toutes les couleurs se fondent sur la description. Ces treize couleurs sont énumérées: louenge, vitupere, allegement, aggregement, magnificacion, depression, supplicacion, exortacion, beneiçon, maudiçon, exhibicion, affection, narracion. Il apparaît ainsi que ces catégories ne sont pas un emprunt au livre IV de l'Ad Herennium sur l'ornatus, les figures de style, mais une adaptation de la doctrine de l'inventio locorum, ou recherche des lieux, des topoï, telle qu'elle est développée dans le De inventione et dans le livre III de l'Ad Herennium. Les deux premières figures de Legrand, louenge et vitupere ou blasme sont les deux formes du genre épidéictique (genus demonstrativum): laus et vituperatio. La description des personnes par louange ou vitupere qu'expose l'auteur repose sur la technique de description à partir des attributa personarum ${ }^{36}$ des deux manuels latins. La description constitue un chapitre important des arts poétiques du XII siècle $^{37}$; et les attributs des personnes, appelés proprietates, attributa ou epitheta personarum lui servent de base. Matthieu de Vendôme, dans son Ars versificatoria (vers 1175) ${ }^{38}$ dresse une liste de onze attributs. Jacques Legrand propose à son élève, comme modèle de base de toute description, un tableau de trente substantifs (p. 86) dans lequel on retrouve, avec amplification de certains éléments, les attributs traditionnels: nomen, natura, fortuna, habitus, etc. Les définitions que J. Legrand donne de chacun de ces substantifs ont un intérêt lexicographique dont il faudra traiter ailleurs. A chacun des trente substantifs qu'il nomme «parties», J. Legrand fait correspondre une série de "dénominations». Ainsi, pour la première partie nature, les

36. De Inventione, I, xxiv-xxv; Ad Herennium, III, vi.

37. Faral, pp. 55-85.

38. Sur Matthieu de Vendôme, voir Faral, pp. 106-193. 
dénominacions sont ame, entendement, memoire, corps, substance (p. 87). Puis il traite des techniques d'abrevatioabregement en conseillant de résumer le discours en quatre points: nature, science, moralité, circonstances. Là encore l'Autorité doit être cherchée, non dans la liste des figures, mais dans la théorie du panégyrique de l'Ad Herennium selon laquelle la louange doit porter sur les circonstances, les qualités physiques et morales: "Laus igitur potest esse rerum externarum, corporis, animi.» ( $A d$ Her., p. 174) Chez Legrand, l'animus devient science et moralité, division bipartite qui correspond au plan du Sophilogium: de amore scientiarum, de amore virtutum.

Avec les couleurs 3, 4, 5, 6 (allegement, aggregement, magnificacion, depression), J. Legrand quitte le domaine de l'éloquence épidéictique pour l'éloquence judiciaire. La transition qu'il opère est ainsi formulée: «Pour ces couleurs, il convient proceder non mie tant seulement par maniere de description mais par maniere de persuasion» (p. 87). L'auteur mentionne que ce sont les principales couleurs, les plus utilisées par les «orateurs, autremens dis advocats» (p. 87). Legrand propose, comme dans l'Ad Herennium (Ad Her., pp. 172-178), quatre types de défense. On sait que dans le modèle latin, la défense peut être fondée sur l'orateur lui-même, sur la personne dont il parle, sur les auditeurs, sur l'action qui est jugée. Legrand aménage ce cadre quadripartite de la manière suivante: l'acteur et sa description, l'action, c'est-à-dire la qualité et l'espèce de péché; la passion, c'est-à-dire le mal subi par la victime; la victime.

Les figures 7 a 12 exposent des techniques de pathos. Il faudra, pour mieux comprendre l'intention de Legrand, pouvoir évaluer sa connaissance de la Rhétorique d'Aristote. Son cuvre conservé comporte des compilations du Stagyrite ${ }^{39}$.

Enfin, la dernière figure, narration, servant selon l'auteur, à «raconter histoire à son propos», ou à «raconter son entencion

39. J. Legrand a compilé un Aristotelis, Senece, Boecii dicta communiora et un Compendium utriusque philosophie sur la métaphysique et la philosophie naturelle d'Aristote. 
a autrui» (p. 122) suit l'enseignement du De inventione au sujet de la narratio. L'orateur peut exposer les faits, mais il peut aussi raconter une anecdote, une fabula ou une historia. Les prédicateurs médiévaux du XIII siècle ont privilégié cet aspect de la rhétorique classique au point d'en tirer un genre autonome: le recueil d'exempla, de même que les maîtres en l'art d'écrire dès le $\mathrm{XI}^{\mathrm{e}}$ siècle avaient créé le recueil de colores rhetorici. Héritier de cette tradition, Jacques Legrand termine sa rhétorique par un recueil d'exempla mythologiques et bibliques auquel il renvoie d'ailleurs pour illustrer cette dernière figure ${ }^{40}$.

De prime abord, le plan de Legrand en figures de pensée et figures de mots semblerait indiquer qu'il entre directement dans la troisième partie de la rhétorique antique: elocutio, le style. Un examen plus attentif révèle que l'auteur se souvient que la première partie inventio, la recherche des idées ou des arguments, est considérée par Cicéron comme la plus importante, valable pour tous les types de discours ${ }^{41}$.

Pourquoi ce glissement dans la définition des figures de pensée, par rapport aux modèles reçus? Nous voyons une explication dans la polysémie du terme "sentence» dans l'AS, référant tour à tour à ce que que la linguistique moderne appelle intention de communication ou message, ce que la théorie du sens, en traductologie, appelle le "vouloir dire" ${ }^{42}$; a la phrase au

40. "[...] mais bien est vray que pour ycelle couleur il est necessité et couvenable de savoir pluseurs hystoires ou fictions des quelles pluseurs sont escriptes a divers propos en ce livre cy present.» (p. 122)

41. Sur l'influence de la doctrine de l'inventio sur la littérature française médiévale, voir D. Kelly, "Topical Invention in Medieval French Literature», in J.J. Murphy, dir. (1978), pp. 231235.

42. M. Lederer et D. Seleskovitch, Interpréter pour traduire (Paris, Didier Érudition, 1984), pp. 22-23. 
sens formel de construction de mots (verba coniuncta) ${ }^{43}$; au sens de la phrase. Notons enfin que sept des figures de pensée de Legrand (5 à 12) entreraient dans le domaine moderne de la pragmatique, décrivant ce que l'on appelle depuis Austin et Searle des actes perlocutoires". La "supplicacion", pour ne donner qu'un exemple, apprend à «encliner volonté d'autrui» ( $p$. 120).

\subsubsection{Les couleurs de parole}

À la différence des couleurs de sentence, les couleurs de parole trouvent leur équivalent parmi les figures de l'Ad Herennium. Une étude comparée des trente-quatre figures latines et des treize couleurs de l'AS permet d'étudier les critères de sélection de l'auteur qui dit avoir choisi les figures «les plus necessaires en françois" (p. 134). Mentionnons que dans la courte introduction à cette rubrique, l'auteur semble hésiter sur la distinction entre sentence et parole; il annonce que les 13 couleurs de son choix sont en réalité des couleurs mixtes «car elles ont leur beauté en sentence et en parole» (p. 134). Il n'est pas indifférent de constater que les couleurs les plus aisément transférables en français portent à la fois sur le fond et la forme. N'est-ce pas une façon de contourner la traditionnelle opposition ad sensum/ad litteram?

L'analyse comparative des figures latines et des couleurs de Legrand nous amène aux remarques suivantes:

1. Legrand suit un principe d'économie: entre figures du même type, il choisit la plus générale et laisse les cas particuliers. Sa première figure est la répéticion, comme la repetitio de l'Ad

43. Ex.: «Par cest exemple tu pues appercevoir comment ceste prose est plus belle pour tant que les sentences sont semblablement terminees, $c^{\prime}$ est assavoir en ir, comme tu pues appercevoir.» (p. 141)

44. J. L. Austin, Quand dire, c'est faire (Paris, Seuil, 1970). J. Searle, les Actes de langage (Paris, Hermann, 1972). 
Herennium, mais il passe les trois suivantes conversio, conplexio, traductio, qui sont des variantes de la première et théoriquement aussi traduisibles en français.

2. Deux aspects sont privilégiés: les stratégies de persuasion (qui ont à voir avec la sentence et c'est sans doute pour cela que l'auteur considère que ces couleurs sont mixtes) et les aspects mélodiques (prosodie).

\section{A) La persuasion}

Ces figures sont quantitativement les plus importantes: $4,5,6$, puis 9 à 13 . Suivent quelques exemples de définition: l'interrogation, c'est «quand nous demandons ce que nous voulons affermer ou ce que nous voulons nier" (p. 135); la correction, «en montrant maniere d'avoir mespris, non obstant que on n'en ait rien mespris» (p. 139); la simulacion, «quant on se excuse de dire ce que l'en dit et quant en disant aucune chose l'en dit que l'en ne l'a mie dit” (p. 139).

\section{B) La prosodie}

La huitième couleur, mesure, requiert toute l'attention de l'auteur. Mesure, que l'auteur définit comme «langage mesuré par certain nombre de syllabes» (p. 136), est l'équivalent de la figure latine compar. Ce point est particulièrement pertinent pour évaluer le passage de l'éloquence latine de Legrand à l'éloquence française. Dans le chapitre "De modis rithmatizandi» de son traité sur les sermons, il définit en effet compar comme: «color [qui] concernit quantitatem pedum seu sillabarum metrorum seu rithmatum ${ }^{45}$. Pour cette couleur qui concerne la substance sonore de la langue, l'auteur précise qu'elle est «moult prouffitable soit en françois soit en latin qui en scet bien user" (p. 136). L'exposé sur cette couleur contient aussi un témoignage précieux sur la traduction de la théorie de la versification. Legrand explique qu'il a choisi de terminer ses phrases par un groupe rythmique de six syllabes et il justifie ce choix en se référant à la métrique latine: "Je cuide que le nombre sizain est

45. Beltran (1967), pp. 162-163. 
le plus convenable, car il ensuit l'accent et la prononciation des vers en latin nomméz exametres.» (p. 136) De la même façon, le second choix devrait être le nombre cinq à cause des penthametres. L'harmonie - c'est le mot qu'utilise l'auteur - est donc directement transférable du latin au français. L'auteur ne semble pas envisager que chacun des systèmes linguistiques possède ses propres règles prosodiques.

\section{8. «Les couleurs pou necessaires»}

Notre connaissance de la liste complète des figures de parole de l'Ad Herennium permet de faire quelques hypothèses sur les raisons du choix de Legrand.

1. Legrand $n^{\prime} a$ pas choisi toutes les figures de raisonnement, pour des raisons que nous ne pouvons élucider à ce stade. Ainsi il omet sententia: usage de maximes tirées de l'expérience, alors que dans l'AS on en compte de nombreuses occurrences.

2. Il renonce a adapter - l'équivalence littérale est impossible - les figures par consonance, portant sur le signifiant latin: similiter cadens, emploi de cas identiques; adnominatio ${ }^{46}$, modification d'un son (durée d'une voyelle, redoublement ou non d'une consonne). Il est vrai que $\mathrm{l}^{\prime} A d$ Herennium réserve ces figures au discours de divertissement.

3. La même remarque s'applique aux figures de construction où l'ornement tient à l'ordre des mots par effet de symétrie ou de dissymétrie: disiunctio, coniunctio, adiunctio, dissolutio, membrum, articulum.

4. Enfin il écarte les figures appartenant à la deuxième partie de la rhétorique: la dispositio (composition du discours): transitio, expeditio.

46. Selon E. Faral (op. cit., pp. 93-97), l'adnominatio, jugée peu importante par l'auteur de l'Ad Herennium, se retrouve fréquemment dans les textes médiévaux en latin et en français. L'auteur donne l'exemple du jeu sur l'amer (amare), l'amer (amarum) et la mer dans le Tristan et dans le Cligès de Chrétien de Troyes. 
La dispositio, importante dans les arts rhétoriques et les artes predicandi, est absente de la rhétorique de l' $A S$. Au chapitre des omissions notables, deux encore doivent être mentionnées. Les tropes, qui occupent habituellement une position intermédiaire dans les recueils entre les figures de mots et les figures de pensée, ne sont pas mentionnées par l'auteur ${ }^{47}$. Et les trois styles latins, simple, tempéré, sublime, ne trouvent pas leur équivalent dans cette rhétorique française ${ }^{48}$.

\section{9. Économie d'ensemble de la rhétorique}

La fonction des couleurs de rhétorique étant élucidée, il est possible de dégager une économie d'ensemble de la rhétorique et de la comparer aux modèles latins. Rappelons que l'AS nous intéresse ici comme exemple de traduction en langue vernaculaire $d^{\prime}$ une théorie du langage et de la communication.

La section sur les couleurs de rhétorique se termine par un chapitre sur les rimes. Selon l'auteur la rime est bien une couleur de rhétorique; il en donne d'ailleurs la définition dans son Introductorium sermocinandi sous l'appellation similiter cadens ou similiter desinens, conformément à l'Ad Herennium ${ }^{49}$. L'auteur lui consacre un développement spécial, «comme celle qui requiert plus grande exposicion» (p. 151). Le contenu de ce chapitre sur

47. Dans les arts poétiques médiévaux, les tropes font partie de l'ornatus difficilis, alors que les autres couleurs appartiennent à l'ornatus facilis. Cf. Faral, pp. 89-97.

48. "Sunt igitur tria genera, quae genera nos figura appellamus, in quibus omnis oratio non vitiosa consumitur: unum gravem, alteram mediocrem, tertiam extenuatam vocamus.) (Ad Her., p. 252). Sur la transmission de cette théorie au Moyen Âge, voir Faral, pp. 86-98.

49. Beltran (1967): "color qui dicitur similiter cadens, aut similiter desinens, qui nomine usuali rithma vocatur» (p. 162). 
les rimes, qui a attiré depuis longtemps l'attention des chercheurs $^{50}$, est celui qui a le moins à voir avec la tradition latine et donc la traduction. J. Legrand y expose les règles de composition des formes métriques françaises du rondeau, de la ballade, du sirventois et du lais. Il $n$ 'en demeure pas moins que la position de ce chapitre dans l'AS s'explique par le cadre théorique latin, les figures 15 et 16 de l'Ad Herennium. L'auteur greffe la Seconde rhétorique en vernaculaire à sa place légitime selon le cadre latin.

Le chapitre sur la mémoire correspond à la quatrième partie de la rhétorique latine: memoria. Les douze règles que Legrand donne à son lecteur sont pour la plupart une application pratique des remarques qu'il a consignées dans son Tractatus de arte memorandi $i^{51}$. Plus que son contenu, c'est la fonction de ce chapitre qui nous intéresse ici comme pièce d'un système théorique.

La dernière partie est appelée par l'auteur: poetrie. Legrand ne donne pas au terme le sens d'art de rimer, comme dans la Poetria nova de Geoffroy de Vinsauf (entre 1208 et 1213) ou la Poetria de Jean de Garlande (non datée; première moitié du $\mathrm{XIII}^{e}$ siècle). Pour l'auteur, poetrie est «science qui aprent a faindre et a faire fictions fondees en raison et en la semblance des choses desquelles on veut parler» (p.147). Cette science est selon lui «subalterne de rhetorique», puisqu'elle sert à alléguer, c'est-àdire à raconter une anecdote pour prouver ou persuader. L'allegacion est «la souveraine couleur car par elle le langage se demonstre meilleur, plus souverain et plus auctentique» (p. 156). La poetrie de Legrand s'articule a la rhétorique comme illustration de la douzième figure de pensée de l'Ad Herennium,

50. Édité par Langlois, op. cit., pp. 1-10.

51. Pour une analyse comparative des deux textes, voir C. le Brun, Les Exemples mythologiques dans l'Archiloge Sophie de jacques Legrand. Analyse et transcription (mémoire de maîtrise inédit, Institut d'études médiévales, Université de Montréal, 1978), pp. $38-48$. 
exemplum: "exemplum est alicuius facti aut dicti praeteriti cum certi auctoris nomine propositio» (Ad Her., p. 382). La position de ce recueil d'exempla - fictions mythologiques et histoires bibliques - à la fin de la rhétorique s'explique de plusieurs façons: par sa longueur; par l'importance qu'accordent les prédicateurs aux exempla; par l'existence de recueils d'exempla autonomes; par le fait que ces exempla sont destinés à être mémorisés par l'orateur et qu'ils sont donc une suite logique des règles de mémoire. De toutes les parties de la rhétorique, la poetrie et son mode d'emploi nous paraît être celle où le texte français traduit deux traditions latines intégrées par l'auteur: l'exemplum de la tradition latine classique et l'exemplum de l'ars predicandi. La résurgence de la composante orale de l'éloquence antique à la fin du Moyen Âge dans la prédication religieuse influence profondément cette rhétorique française qui se veut un art de la communication orale plus qu'une stylistique à la manière des arts poétiques ou des artes dictaminis. Notre analyse de la rhétorique de l'AS comme traduction d'un modẻle théorique latin est illustrée dans le tableau I.

3. Les choix et les techniques de traduction de Jacques Legrand

3.1. Introduction de nouveaux concepts et signifiants et adaptation à la culture-cible

Nos observations se regroupent sous deux rubriques: l'adaptation de l'énoncé et l'adaptation de l'énonciation.

\subsubsection{Adaptation de l'énoncé}

Pour introduire le lexique théorique de ce catalogue des figures de style, l'auteur utilise une gamme de procédés que l'on peut résumer ainsi: 
1) Utilisation de binômes synonymiques ${ }^{52}$ pour fins d'explicitation conceptuelle, binômes reliés par ou (équivalence) ou par et (approximation, analogie). Exemples: vitupere ou blasme; alleguier ou appliquier; moz et denominacions; magnifier et louer; desprisier et appeticier; allegier et supporter le mefait; mesprendre et pechier.

2) Renvoi au terme d'usage courant dans la culture-cible par les locutions «autrement dit» et "c'est assavoir». Ex.: «orateurs autrement dis avocats» (hypotaxe).

3) Définition des nouveaux termes caractérisée par

a) l'emploi du verbe correspondant au substantif défini, ce verbe étant lui-même souvent explicité dans un binôme synonymique («magnification, la quelle apprent et demonstre comment on doit le bien d'aucune ame magnifier et aggrandir» (p. 119)); ou de l'adjectif ("opposicion, quant on parle de deux choses opposites en comparant l'une a l'autre» (p. 137));

b) la présence $d^{\prime}$ un groupe synonyme du substantif défini («exclamacion, la quelle se fait quant nous parlons par grant admiration et par une maniere de escry» (p. 134));

c) le renvoi à la fonction ou au mode d'emploi de la figure. Fonction: «exhibicion, la quelle aprent comment on se puet offrir a autrui» (p. 121). Mode d'emploi: «continuacion, la quelle se fait quant en nostre en nostre langage nous mectons plusieurs moz et plusieurs sentences l'une apres l'autre» (p. 136). La définition par la fonction correspond aux figures de pensée alors que que la définition par le mode d'emploi est réservée aux figures de parole.

4) L'illustration par de nombreux exemples. Ex. dans l'exposé sur le panégyrique selon les trente attributs, pour l'attribut «science»: «Par science nous povons dire ainsi: tu es

52. Sur les binômes synonymiques en moyen français, voir $\mathrm{C}$. Buridant, «les Binômes synonymiques. Esquisse d'une histoire des couples de synonymes du Moyen Âge au XVII' siècle», Bulletin du Centre d'analyse du discours 4 (1980), pp. 5-80. 
clerc, sage et bien apris, et de toutes les sciences tu as la congnoissance; tu sais bel parler par rethorique» (p. 90).

\subsubsection{Adaptation de l'énonciation}

La comparaison de la rhétorique de l'AS avec l'Introductorium sermocinandi, traité latin de l'auteur sur un sujet appartenant au domaine de la rhétorique, fait ressortir les différences suivantes:

1) La grande visibilité de l'énonciateur dans le texte français; sa présence n'est qu'implicite en latin par des adjectifs évaluatifs. Dans l'AS - et surtout dans la rhétorique - on relève de nombreuses occurrences du «je» et des variantes: «a mon avis», «m'est avis que». Nous notons aussi l'association de la première personne à des formes de l'accompli (ex. «j'ai expérimenté ${ }^{53}$ ).

2) L'inscription répétitive du destinataire, qui est beaucoup plus présent dans la rhétorique que dans le reste de $l^{\prime} A S$. Aux formes impersonnelles de l'adjectif verbal (advertendum, cavendum, loquendum) et aux rares occurrences de la deuxième personne dans le style formulaire «si velis», correspond le «tu», souvent accompagné des modalisateurs devoir et pouvoir. Ex: «tu dois aviser que». La formule de liaison par excellence est: «oultre plus dois savoir que». Enfin l'usage de la deuxième personne est lié à des énoncés perlocutoires du type: "comme tu peux clairement apercevoirs ${ }^{54}$.

53. Dans la présentation des règles de mémoire: «mais des anciens je prenray aucune racine et si scripray ce que j'ay experimenté en moy" (p. 145).

54. Ex.: "comme tu pues clerement appercevoir"; "comme tu pues appercevoir en parlant» (p. 137). 
3) L'emploi du nous inclusif ${ }^{55}$ dans le texte français, renvoyant à la culture partagée, celle des locuteurs français. Ex: «comme nous disons en notre françois».

\subsection{Le traducteur et l'autorité: Jacques Legrand traducteur de Pierre Bersuire}

Les exempla de J. Legrand sont de deux types: mythologiques et bibliques. Evencio Beltran a identifié exactement le texte et même le manuscrit utilisés par Legrand comme source des exempla mythologiques: les «fictions Ovide» ${ }^{56}$. Comme nous l'avons mentionné plus haut, Legrand a utilisé l'Ovidius moralizatus, livre $\mathrm{XV}$ du Repertorium morale de Pierre Bersuire. Pour rappeler en quelques mots la nature de l'Ovidius moralizatus, qu'il suffise de mentionner qu'à partir du XII siècle, les commentaires d'Ovide se multiplient, visant à découvrir sous la surface de la fable antique des vérités conformes à la Révélation chrétienne. Dans le même temps, des traités de mythographie sont consacrés à $l^{\prime}$ interprétation des attributs et des faits des dieux antiques en terme de vices et de vertus. Le travail de Bersuire consiste donc en un portrait des dieux et un récit de chacune des métamorphoses d'Ovide suivis d'une interprétation morale et allégorique ${ }^{57}$.

Legrand suit le plan de Bersuire dans son recueil de «fictions Ovide». Mais comme son propos est de raconter ces fictions «en brief» (p. 156), il use, en traduction, d'une technique

55. Le «nous» pourrait parfois exclure le destinataire. Ex.: «latin, tierce principale, de la quelle nous usons» (p. 64).

56. E. Beltran, «Une source de l'Archiloge Sophie de Jacques Legrand: l'Ovidius moralizatus de Pierre Bersuire», Romania 100 (1979), pp. 483-501.

57. Cf. le prologue du Repertorium morale: "Non intendo nisi rarissime litteralem sensum fabularem tangere, sed solum circa expositionem moralem et allegoricam laborare.» (cité par J. Seznec, 1972, p. 93) 
qui semble caractériser sa méthode de travail: l'abrevatio, l'abrégé. On peut dire, en reprenant les catégories de Jakobson ${ }^{58}$, que Legrand procède de la même façon en traduction interlinguale qu'en traduction intralinguale. De la somme au manuel, voilà une formule qui nous semble caractériser l'activité intellectuelle de Legrand.

La technique de traduction de l'Ovidius moralizatus peut se décrire de la façon suivante.

1) Legrand garde le récit et omet le commentaire. Il sélectionne dans le récit de Bersuire des membres de phrase qu'il traduit littéralement et articule par des charnières peu variées: un «et» à valeur additive et temporelle ${ }^{59}$, un «dont» consécutif et un «lequel» traduisant le relatif de liaison (voir tableau II).

2) Il donne à chacune des fictions un titre. Celui-ci constitue, explique-t-il, un mot-vedette qui permettra à l'utilisateur du manuel de repérer rapidement les histoires correspondant au thème qui l'intéresse (p. 157). Les passages sélectionnés le sont donc en fonction du titre prévu. Alléguer, on l'a vu, c'est appliquer une histoire à son propos.

3) Les titres résument la teneur d'une des interprétations de Bersuire. Si la majorité des titres choisissent l'interprétation morale, un certain nombre renvoient à l'interprétation allégorique. Le combat d'Hercule et d'Antée, par exemple, où ce dernier tire sa force du contact avec la terre (IX,11), est intitulé humilité. Ce qui renvoie à Bersuire: «tactum terrae id est

58. Essais de linguistique générale (Paris, Éditions de Minuit, 1963), ch. 4.

59. Selon les termes de C. Buridant, le "et» est «le strument polysémique de l'ancien français». Cf. «Blaise de Vigenère traducteur de "la Conquête de Constantinople» de Geoffroy de Villehardouin", Reoue des sciences humaines 180 (Lille, 1980), p. 115. 
humilitatem uel propriae fragilitatis memoriam ${ }^{60}$. Quelques titres sont doubles, qui renvoient à deux interprétations de Bersuire: l'épisode du tonneau de Danaé est intitulé: AvariceMarie. Le tableau III donne quelques autres illustrations. Alors que les Ovides moralisés et notamment celui de Bersuire déploient les multiples significations cachées sous la lettre, sous l'écorce de la fabula, Legrand impose par sa présélection du texte a traduire une lecture monosémique. Où d'autres ont glosé, Legrand abrège. L'enjeu est-il de retrouver la lettre et l'esprit du texte primitif? Il nous faut malheureusement répondre par la négative. Malgré ses choix humanistes, malgré sa prédilection pour les poètes antiques, il assujettit les Métamorphoses en en faisant des exempla à forte orientation morale. L'analyse statistique des 400 titres $^{61}$ mène à la conclusion suivante: $\mathrm{Si}$ quelques titres résistent à toute moralisation et semblent être choisis seulement pour le plaisir de la fabula (beauté, habondance, jeu, marrastre, medecine, moustre, etc.), la moitié d'entre eux stigmatisent des vices et des péchés, l'orgueil et la luxure en tête. Sous cet angle, l'AS rejoint les miroirs du prince à orientation plus éthique que politique, notamment le Livre de bonnes meurs, l'autre traité français de Legrand.

L'attitude de Legrand vis-à-vis du texte-source est sans équivoque: Bersuire, commentateur d'Ovide, n'est qu'un maillon dans la chaîne de la transmission, un outil de travail. Il n'a pas le statut d'autorité et ne mérite donc pas ce que Peter Dembrovsky appelle la traduction-service (service translation) ${ }^{62}$.

60. Ed. J. Engels, Petrus Berchorius, Reductorium Morale, lib. XV «Ovidius Moralizatus» (Utrecht, 1962, 1966), p. 141.

61. Voir le Brun, pp. 62-79.

62. P. Dembowski, «Learned Latin Treatises in French: Inspiration, Plagiarism, and Translation", Viator 17 (1986), pp. 255-266. Sur cette question, voir aussi A. J. Minnis, Medieval Theory of Authorship. Scolastic Literary Attitudes in the Later Middle Ages (London, Scholar Press, 1984); J. Blanchard, "Compilation et légitimation au XVe siècle», Poétique 18 (1988), pp. 139-157. 
Face au texte-source, Legrand semble être sélectif par souci de pédagogie et littéral par commodité ou efficacité.

\section{Conclusion}

Nous nous en tiendrons à quelques conclusions partielles et provisoires sur cette rhétorique de l'AS où il reste encore des trésors enfouis à découvrir, pour reprendre un topos récurrent sur la transmission des connaissances au Moyen $\hat{A} g \mathrm{e}^{63}$.

Jacques Legrand, clerc, homme politique, est orateur en latin et en français; cet état de diglossie l'autorise à fixer les frontières entre le transférable et le non-transférable en français. La culture-source latine est accessible au seul clerc, la culturecible est une culture partagée.

L'épreuve de la traduction amène l'auteur à repenser les catégories du modèle théorique et notamment la distinction traditionnelle entre les figures de pensée et les figures de mots.

Le transférable de la rhétorique classique consiste en des stratégies de persuasion que le prince saura utiliser à des fins diplomatiques et en des canons esthétiques: l'auteur définit une harmonie basée sur le rythme et la répétition.

Dans sa traduction-adaptation française, J. Legrand se constitue en Autorité. Nous avons observé le changement du point de vue énonciatif et le traitement sélectif du texte de Bersuire. L'auteur, qui annonce qu'il prendra «aucune racine» des Anciens ${ }^{64}$, manifeste une grande liberté dans le choix des contenus et l'expression des opinions.

Aussi, en définitive, Jacques Legrand apparaît-il plus auteur dans ce manuel-traduction en vernaculaire, où il établit

63. Cf. E. Curtius, la Littérature européenne et le Moyen Age latin (Paris, P.U.F., 1956), p. 108.

64. Voir note 55. 
une relation pédagogique avec le destinataire, que dans l'ensemble de ses travaux en latin où il se plie aux modèles du florilège, du compendium ou du commentaire.

Université Concordia 


\begin{tabular}{||ll||}
\hline Couleurs de sentence & Inventio \\
La description; les XIII couleurs de & (De Inventione, I, II; Ad \\
sentence et leur mode d'emploi: & Herennium, III; De \\
1. louenge & differentiis topicis; Rhetorica, \\
2. vitupere ou blasme & I) \\
3. allegement & \\
4. aggregement & \\
5. magnificacion & \\
6. depression & \\
7. supplicacion & \\
8. exortacion & \\
9. beneiçon & \\
10. maudiçon & \\
11. exhibicion & \\
12. affection & \\
13. narracion. & \\
\hline Couleurs de parole et autres & Elocutio \\
couleurs de thétorique & (Ad Herennium, IV): \\
Les XIII couleurs de parole: & exornationes verborum \\
1. repeticion & repetitio (1) \\
2. gradacion & gradatio (19) \\
3. exclamacion & exclamatio (6) \\
4. interrogacion & interrogatio (4) \\
5. opposicion & contentio (5) \\
6. resonnement & ratiocinatio (8) \\
7. continuacion & continuatio (13) \\
8. mesure & compar (14) \\
9. premission & conclusio (35) \\
10. certifficacion & permissio (30) \\
11. correction & correctio (22) \\
12. simulacion & occultatio (23) \\
13. excitacion. & conduplicatio (27) \\
- les rimes & similiter cadens, desinens \\
& (15,16) \\
\hline Reggles de mémoire & Memoria \\
\hline Poetrie et allegacion & exemplum (Ad Her., IV, \\
& exornationes sententiarum, \\
\hline Recueil d'exempla mythologiques & I2) \\
et bibliques & Inventio, Dispositio, \\
& Elocutio. Artes predicandi: \\
\hline & Recueil d'exempla \\
\hline
\end{tabular}


TABLEAU II: TECHNIQUE DE TRADUCTION DE L'OVIDIUS MORALIZATUS DE BERSUIRE (DE FORMIS FIGURISQUE DEORUM)

\begin{tabular}{|c|c|}
\hline Pierre Bersuire & Jacques Legrand \\
\hline $\begin{array}{l}\text { (...) de Saturno qui pater et } \\
\text { primus predicatur esse deorum } \\
\text { (...). Saturnus ergo pingebatur et } \\
\text { supponebatur esse homo senex, } \\
\text { curvus, tristis et pallidus, } \\
\text { qui una manu falcem tenebat et } \\
\text { in eadem portabat drachonis } \\
\text { ymaginem qui dentibus caudam } \\
\text { propriam commordebat, }\end{array}$ & $\begin{array}{l}\quad \text { Dissimulacion } \\
\text { Saturnus est le plus ancien des } \\
\text { dieux et est paint viel, triste et } \\
\text { pale. } \\
\quad \text { Fausseté } \\
\text { Saturne a une main tenoit une } \\
\text { faucille et portoit le dragon } \\
\text { mordant sa queue }\end{array}$ \\
\hline $\begin{array}{l}\text { altera vero filium parvulum ad } \\
\text { os applicabat et eum propriis } \\
\text { dentibus devorabat. Quatuor } \\
\text { iuxta se habebat liberos s. } \\
\text { Iovem, Iunonem, Neptunum et } \\
\text { Plutonem }\end{array}$ & \begin{tabular}{l}
\multicolumn{1}{c}{ Cruauté } \\
Saturne, a l'autre main, tenoit un \\
enfant et le devoroit de ses \\
propres dens, presens ses .iij. \\
enfans, c'est assavoir Jupiter, \\
Junon, Neptune et Pluton.
\end{tabular} \\
\hline $\begin{array}{l}\text { quorum Iupiter patris virilia } \\
\text { amputabat. Mare eciam ante } \\
\text { eum pictum erat in quo vid. } \\
\text { dicta Saturni scissa virilia } \\
\text { proiecta videbantur, de quibus } \\
\text { Venus puella pulcherrima } \\
\text { nascebatur. }\end{array}$ & $\begin{array}{l}\text { Luxure } \\
\text { Jupiter coupa les genitaires a } \\
\text { Saturne son pere et les gecta en } \\
\text { la mer, dont fut Venus nee, tres } \\
\text { belle pucelle. }\end{array}$ \\
\hline
\end{tabular}


TABLEAU III: EXPLICATION DES TITRES

\begin{tabular}{||l|l|l||}
\hline Dissimulacion & $\begin{array}{l}\text { Saturnus est le plus } \\
\text { ancien des dieux et est } \\
\text { paint viel, triste et pale. }\end{array}$ & $\begin{array}{l}\text { Capite velatus quia bona } \\
\text { debet per humilitatem } \\
\text { abscondere et non } \\
\text { publice propalare. }\end{array}$ \\
\hline Fausseté & $\begin{array}{l}\text { Saturne a une main tenoit } \\
\text { une faucille et portoit le } \\
\text { dragon mordant sa } \\
\text { queue. }\end{array}$ & $\begin{array}{l}\text { Falcem tenentem i. } \\
\text { falsitate et malicia } \\
\text { depravatum. }\end{array}$ \\
\hline Luxure & $\begin{array}{l}\text { Jupiter coupa les } \\
\text { genitaires a Saturne et les } \\
\text { gecta en la mer, dont } \\
\text { Venus fut nee, tres belle } \\
\text { pucelle. }\end{array}$ & $\begin{array}{l}\text { Venus enim in mari } \\
\text { fingitur genita pro eo } \\
\text { quod luxuria ab } \\
\text { opulentia et a deliciarum } \\
\text { fluxibus non recedit. }\end{array}$ \\
\hline Justice & $\begin{array}{l}\text { Appollo une serpent de } \\
\text { sa saiette perçoit et a } \\
\text { mort mectoit. }\end{array}$ & $\begin{array}{l}\text { Arcu, pharetra et sagitta } \\
\text { lusticie armatus et ad } \\
\text { sagittandum, puniendum } \\
\text { et corrigendum Phitonem } \\
\text { id est serpentinos } \\
\text { peccatores debet esse pre } \\
\text { ceteris adornatus. }\end{array}$ \\
\hline Orgueil & $\begin{array}{l}\text { Mercure en sa main avoit } \\
\text { eles et de serpens } \\
\text { environnez estoit et un } \\
\text { coutel corbe tenoit. }\end{array}$ & $\begin{array}{l}\text { Pedes et caput alatos per } \\
\text { presumptionem et } \\
\text { superbiam. }\end{array}$ \\
\hline
\end{tabular}

